PROCEEDINGS OF THE

AMERICAN MATHEMATICAL SOCIETY

Volume 139, Number 2, February 2011, Pages 401-405

S 0002-9939(2010)10694-9

Article electronically published on September 21, 2010

\title{
GENERATING THE INFINITE SYMMETRIC GROUP USING A CLOSED SUBGROUP AND THE LEAST NUMBER OF OTHER ELEMENTS
}

\author{
J. D. MITCHELL, M. MORAYNE, AND Y. PÉRESSE
}

(Communicated by Julia Knight)

\begin{abstract}
Let $S_{\infty}$ denote the symmetric group on the natural numbers $\mathbb{N}$. Then $S_{\infty}$ is a Polish group with the topology inherited from $\mathbb{N}^{\mathbb{N}}$ with the product topology and the discrete topology on $\mathbb{N}$. Let $\mathfrak{d}$ denote the least cardinality of a dominating family for $\mathbb{N}^{\mathbb{N}}$ and let $\mathfrak{c}$ denote the continuum. Using theorems of Galvin, and Bergman and Shelah we prove that if $G$ is any subgroup of $S_{\infty}$ that is closed in the above topology and $H$ is a subset of $S_{\infty}$ with least cardinality such that $G \cup H$ generates $S_{\infty}$, then $|H| \in\{0,1, \mathfrak{d}, \mathfrak{c}\}$.
\end{abstract}

The symmetric group $S_{\infty}$ is a Polish group under the topology inherited from the product topology on $\mathbb{N}^{\mathbb{N}}$ with the discrete topology on $\mathbb{N}$; see [8, Section $9 . \mathrm{B}(7)$ ] for further details. We will refer to subgroups $G$ of $S_{\infty}$ that are closed in this topology as closed subgroups. It is a well-known fact that the closed subgroups of $S_{\infty}$ are precisely the automorphism groups of relational structures on $\mathbb{N}$; see, for example, 4. Theorem 5.8]. Such automorphism groups have been widely investigated; see for example [9] and the references therein.

The theorem of Bergman and Shelah from [3] on which our main theorem (Theorem 1.3) is based involves the following equivalence relation $\approx$ on subgroups of $S_{\infty}$. Let $G$ and $H$ be (not necessarily closed) subgroups of $S_{\infty}$. Then $G \approx H$ if there exists a finite $F \subseteq S_{\infty}$ such that the subgroup $\langle H, F\rangle$ generated by $H$ and $F$ equals $\langle G, F\rangle$.

Throughout, we write functions to the left of their argument and compose from right to left. The following two subgroups of $S_{\infty}$ are representatives of two of the classes under $\approx$. Let $A$ be a partition of $\mathbb{N}$ into sets $A_{1}, A_{2}, \ldots$ where $\left|A_{i}\right|=i$ for all $i \geq 1$ and let $B$ be a partition of $\mathbb{N}$ into sets $B_{0}, B_{1}, \ldots$ with $\left|B_{i}\right|=2$ for all $i \in \mathbb{N}$. Then define

$$
\begin{aligned}
H_{\mathbb{N}} & =\left\{f \in S_{\infty}: f\left(A_{i}\right)=A_{i} \text { for all } i \geq 1\right\}, \\
H_{2} & =\left\{f \in S_{\infty}: f\left(B_{i}\right)=B_{i} \text { for all } i \in \mathbb{N}\right\} .
\end{aligned}
$$

It is straightforward to verify that $H_{\mathbb{N}}$ and $H_{2}$ are closed subgroups of $S_{\infty}$. If $G$ is a subgroup of $S_{\infty}$ and $\Sigma \subseteq \mathbb{N}$, then the pointwise stabilizer of $\Sigma$ in $G$ is the subgroup $G_{(\Sigma)}=\{f \in G: f(\sigma)=\sigma$ for all $\sigma \in \Sigma\}$. We require the following slightly weaker version of the main theorem in Bergman and Shelah [3].

Received by the editors February 4, 2010.

2010 Mathematics Subject Classification. Primary 20B07; Secondary 54H11.

(C)2010 American Mathematical Society 
Theorem 1.1 (Bergman and Shelah [3]). Let $G$ be a closed subgroup of $S_{\infty}$. Then exactly one of the following holds:

(i) $G_{(\Sigma)}$ has an infinite orbit for all finite $\Sigma \subseteq \mathbb{N}$ and $G \approx S_{\infty}$;

(ii) $G_{(\Sigma)}$ has only finite orbits for some finite $\Sigma \subseteq \mathbb{N}, G_{(\Gamma)}$ has orbits of unbounded length for all finite $\Gamma \subseteq \mathbb{N}$, and $G \approx H_{\mathbb{N}}$;

(iii) $G_{(\Sigma)}$ has orbits of bounded length for some finite $\Sigma \subseteq \mathbb{N}$ and $G \approx H_{2}$ or $G \approx\left\{1_{\mathbb{N}}\right\}$.

It is also shown in 3 that no two of the groups $\left\{1_{\mathbb{N}}\right\}, H_{2}, H_{\mathbb{N}}$, and $S_{\infty}$ are equivalent under $\approx$.

Mesyan 13 considered the natural analogue of $\approx$ on the closed subsemigroups of the semigroup $\mathbb{N}^{\mathbb{N}}$. The situation is much more complicated in $\mathbb{N}^{\mathbb{N}}$, as in particular, there are infinitely many distinct equivalence classes in this setting. However, if the class of closed subsemigroups considered is restricted to endomorphism semigroups of bipartite graphs or partial orders on $\mathbb{N}$, then again a classification can be achieved. In particular, in [14] it is shown that such semigroups lie in precisely two equivalence classes under the analogue of $\approx$. In 12 . Mesyan shows that certain closed subrings of the direct sum of $\aleph_{0}$ many copies of a simple ring fall into two distinct equivalence classes under the appropriate analogue of $\approx$.

Macpherson and Neumann [11, Theorem 1.1] showed that $S_{\infty}$ is not the union of a countable chain of proper subgroups. Thus if $G$ is any subgroup of $S_{\infty}$ and $H \subseteq S_{\infty}$ with least cardinality such that $\langle G, H\rangle=S_{\infty}$, then $|H| \neq \aleph_{0}$. The following theorem of Galvin implies that, in fact, $|H| \leq 1$ or $|H|>\aleph_{0}$.

Theorem 1.2 (Theorem 5.8 in Galvin [6]). Let $G$ be subgroup of $S_{\infty}$ such that there exists a countable $H \subseteq S_{\infty}$ with $\langle G, H\rangle=S_{\infty}$. Then there exists $f \in S_{\infty}$ such that $\langle G, f\rangle=S_{\infty}$.

So, as Bergman and Shelah comment in [3, there is a gap between those subgroups of $S_{\infty}$ over which it is 'easy' to generate $S_{\infty}$ and those where it is 'difficult'. The aim of this paper is, in some sense, to make this comment more precise in the case of closed subgroups of $S_{\infty}$.

If $G$ is a subgroup of the symmetric group $S_{\infty}$, then, for the sake of brevity, we will denote by $\operatorname{rank}\left(S_{\infty}: G\right)$ the least cardinality of a subset $H$ of $S_{\infty}$ such that the group generated by $G \cup H$ equals $S_{\infty}$. By Theorem $1.2 \operatorname{rank}\left(S_{\infty}: G\right) \leq 1$ if and only if $S_{\infty} \approx G$. Also if $G \not \approx S_{\infty}$ and $H$ is a subgroup of $S_{\infty}$ such that $G \approx H$, then $\operatorname{rank}\left(S_{\infty}: G\right)=\operatorname{rank}\left(S_{\infty}: H\right)>\aleph_{0}$. The cardinal $\operatorname{rank}\left(S_{\infty}: G\right)$ will be referred to as the relative rank of $G$ in $S_{\infty}$. Relative ranks have been studied in the context of semigroups; see 5, 7], or [14 for further details.

A subset $D$ of $\mathbb{N}^{\mathbb{N}}$ satisfying the following condition is called a dominating family for $\mathbb{N}^{\mathbb{N}}$ :

for all $f \in \mathbb{N}^{\mathbb{N}}$ there exists $g \in D$ such that $f(n) \leq g(n)$ for all $n \in \mathbb{N}$.

The least cardinality of a dominating family for $\mathbb{N}^{\mathbb{N}}$ is denoted by $\mathfrak{d}$, and the continuum is denoted by $\mathfrak{c}$. It is clear that $\aleph_{1} \leq \mathfrak{d} \leq \mathfrak{c}$. If the continuum hypothesis holds, then $\aleph_{1}=\mathfrak{d}=\mathfrak{c}$. However, the following theories are also consistent: ZFC + $\left(\mathfrak{d}=\aleph_{1}<\mathfrak{c}\right)$ and ZFC $+\left(\aleph_{1}<\mathfrak{d}=\aleph_{2}=\mathfrak{c}\right)$; see [2] for further details.

The following is the main theorem of this paper.

Theorem 1.3. Let $G$ be a closed subgroup of $S_{\infty}$. Then exactly one of the following holds: 
(i) $G_{(\Sigma)}$ has an infinite orbit for all finite $\Sigma \subseteq \mathbb{N}$ and $\operatorname{rank}\left(S_{\infty}: G\right) \in\{0,1\}$;

(ii) $G_{(\Sigma)}$ has only finite orbits for some finite $\Sigma \subseteq \mathbb{N}, G_{(\Gamma)}$ has orbits of unbounded length for all finite $\Gamma \subseteq \mathbb{N}$, and $\operatorname{rank}\left(S_{\infty}: G\right)=\mathfrak{d}$;

(iii) $G_{(\Sigma)}$ has orbits of bounded length for some finite subset $\Sigma$ of $\mathbb{N}$ and $\operatorname{rank}\left(S_{\infty}: G\right)=\mathfrak{c}$.

Proof. (i). By assumption and by Theorem 1.1, $G \approx S_{\infty}$ and so, by Theorem 1.2. it follows that $\operatorname{rank}\left(S_{\infty}: G\right) \in\{0,1\}$.

(ii). Again by assumption and by Theorem 1.1, $G \approx H_{\mathbb{N}}$ and so $\operatorname{rank}\left(S_{\infty}: G\right)=$ $\operatorname{rank}\left(S_{\infty}: H_{\mathbb{N}}\right)$. Hence it suffices to $\operatorname{prove}$ that $\operatorname{rank}\left(S_{\infty}: H_{\mathbb{N}}\right)=\mathfrak{d}$.

We start by showing that $\operatorname{rank}\left(S_{\infty}: H_{\mathbb{N}}\right) \geq \mathfrak{d}$. By Tychonoff's Theorem 8 , Proposition 4.1(vi)], $H_{\mathbb{N}}$ is compact. Let $U \subseteq S_{\infty}$ be such that $\left\langle H_{\mathbb{N}}, U\right\rangle=S_{\infty}$. Since $H_{\mathbb{N}} \not \approx S_{\infty}$, it follows that $|U|>\aleph_{0}$. The group $\left\langle H_{\mathbb{N}}, U\right\rangle$ is the union of the sets $H_{\mathbb{N}} u_{n} H_{\mathbb{N}} u_{n-1} \cdots u_{0} H_{\mathbb{N}}$, where $u_{0}, u_{1}, \ldots, u_{n} \in U \cup U^{-1}$ (where $U^{-1}=\left\{u^{-1}\right.$ : $u \in U\}$ ). Since $H_{\mathbb{N}}$ is compact, right multiplication in any topological group is continuous, and the continuous image of a compact set is compact, it follows that each $H_{\mathbb{N}} u_{i}$ is compact. Hence, as the product of compact sets in a topological group is compact [1, Proposition 1.4.31], $H_{\mathbb{N}} u_{n} H_{\mathbb{N}} u_{n-1} \cdots u_{0} H_{\mathbb{N}}$ is compact. In other words, $\left\langle H_{\mathbb{N}}, U\right\rangle$ is the union of $|U|$ compact sets. It is straightforward to prove that the minimum number of compact subsets covering $\mathbb{N}^{\mathbb{N}}$ is $\mathfrak{d}$. Hence, since $S_{\infty}$ and $\mathbb{N}^{\mathbb{N}}$ are homeomorphic by [8, Theorem 7.7], it follows that the minimum number of compact subsets covering $S_{\infty}$ is $\mathfrak{d}$. Thus $|U| \geq \mathfrak{d}$ and $\operatorname{so} \operatorname{rank}\left(S_{\infty}: H_{\mathbb{N}}\right) \geq \mathfrak{d}$.

We will now prove that $\operatorname{rank}\left(S_{\infty}: H_{\mathbb{N}}\right) \leq \mathfrak{d}$. Let $M=\left\{m_{0}, m_{1}, \ldots\right\}$ be any subset of $\mathbb{N}$ with $|M|=|\mathbb{N} \backslash M|=|\mathbb{N}|$. Then, by Bergman and Shelah's Theorem[1.1(i), the pointwise stabilizer $S_{(\mathbb{N} \backslash M)}$ of $\mathbb{N} \backslash M$ in $S_{\infty}$ satisfies $S_{(\mathbb{N} \backslash M)} \approx S_{\infty}$. Hence it suffices to prove that there exists $X \subseteq S_{\infty}$ with $|X|=\mathfrak{d}$ such that $S_{(\mathbb{N} \backslash M)} \subseteq\left\langle H_{\mathbb{N}}, X\right\rangle$.

Let $D$ be any dominating family for $\mathbb{N}^{\mathbb{N}}$ with $|D|=\mathfrak{d}$. Replacing any $f \in D$ by $f^{\prime} \in \mathbb{N}^{\mathbb{N}}$ defined recursively by setting $f^{\prime}(0)=f(0)+2$ and

$$
f^{\prime}(i)=\max \left\{f^{\prime}(i-1)+1, f(i)\right\}
$$

for all $i>0$, we may assume that $D$ consists entirely of strictly increasing functions $f^{\prime}$ where $f^{\prime}(i) \geq 2$ for all $i \in \mathbb{N}$. We denote by $T(f)$ the set of all involutions $g \in S_{(\mathbb{N} \backslash M)}$ (elements of order 2) such that $g\left(m_{i}\right) \in\left\{m_{0}, m_{1}, \ldots, m_{f(i)}\right\}$ for all $i \in \mathbb{N}$. Since $D$ is a dominating family, $\bigcup_{f \in D} T(f)$ is the set of all involutions in $S_{(\mathbb{N} \backslash M)}$. The symmetric group $S_{\infty}$ is generated by its involutions [6, Lemma 2.2], and so certainly $S_{(\mathbb{N} \backslash M)}$ is also generated by its involutions. Hence

$$
\left\langle\bigcup_{f \in D} T(f)\right\rangle=S_{(\mathbb{N} \backslash M)} .
$$

We will prove that for all $f \in D$ there exist $g_{f}, h_{f} \in S_{\infty}$ such that $T(f) \subseteq$ $\left\langle H_{\mathbb{N}}, g_{f}, h_{f}\right\rangle$. Consequently,

$$
S_{(\mathbb{N} \backslash M)}=\left\langle\bigcup_{f \in D} T(f)\right\rangle \subseteq\left\langle H_{\mathbb{N}} \cup\left\{g_{f}, h_{f}: f \in D\right\}\right\rangle .
$$

$\operatorname{Thus} \operatorname{rank}\left(S_{\infty}: H_{\mathbb{N}}\right) \leq|D|=\mathfrak{d}$.

Let $f \in D$ be arbitrary and let $N$ be any subset of $\mathbb{N} \backslash M$ such that $|N|=\mid \mathbb{N} \backslash[M \cup$ $N]|=| \mathbb{N} \mid$. Partition $N$ into sets $N_{0}, N_{1}, \ldots$ where $N_{i}=\left\{n_{i, 0}, n_{i, 1}, \ldots, n_{i, f(i)}\right\}$ and define $N_{i}^{*}=\left\{n_{r, i}, n_{r+1, i}, \ldots, n_{f(i), i}\right\}$ where $r=\min \{j \in\{0,1, \ldots, f(i)\}: i \leq f(j)\}$. 
The subgroup $H_{\mathbb{N}}$ was defined as

$$
H_{\mathbb{N}}=\left\{g \in S_{\infty}: g\left(A_{i}\right)=A_{i} \text { for all } i \geq 1\right\},
$$

where $A$ is a partition of $\mathbb{N}$ into sets $A_{1}, A_{2}, \ldots$ satisfying $\left|A_{i}\right|=i$ for all $i \geq 1$. Hence, since $f(i) \geq 2$ for all $i \in \mathbb{N}$, it follows that $f(i)+2 \leq\left|A_{2 f(i)}\right|=2 f(i)$. Since the complements of $M \cup N$ and $\bigcup_{i \in \mathbb{N}} A_{2 f(i)}$ in $\mathbb{N}$ are infinite, there exist $g_{f}, h_{f} \in S_{\infty}$ such that

$$
g_{f}\left(N_{i} \cup\left\{m_{i}\right\}\right) \subseteq A_{2 f(i)} \text { and } h_{f}\left(N_{i}^{*} \cup\left\{m_{i}\right\}\right) \subseteq A_{2 f(i)}
$$

for all $i \in \mathbb{N}$.

Let $t \in T(f)$ be arbitrary and let $k \in S_{\infty}$ be such that $t\left(m_{i}\right)=m_{k(i)}$ for all $i \in \mathbb{N}$. Note that $k$ is an involution since $t$ is an involution. It follows from the definition of $T(f)$ that $k(i) \leq f(i)$ for all $i \in \mathbb{N}$ and so the element $n_{i, k(i)}$ exists for all $i \in \mathbb{N}$. Hence there exist involutions $p, q \in S_{\infty}$ such that $p$ swaps $m_{i}$ and $n_{i, k(i)}$ for all $i \in \mathbb{N}$ with $i<k(i), q$ swaps $m_{k(i)}$ and $n_{i, k(i)}$ for all $i \in \mathbb{N}$ with $i<k(i)$, and all other elements are fixed pointwise by $p$ and $q$.

Since $m_{i}, n_{i, k(i)} \in N_{i} \cup\left\{m_{i}\right\}$, we have that $g_{f}\left(m_{i}\right), g_{f}\left(n_{i, k(i)}\right) \in A_{2 f(i)}$ for all $i \in \mathbb{N}$. Hence the involution $g_{f} p g_{f}^{-1}$, which swaps $g_{f}\left(m_{i}\right)$ and $g_{f}\left(n_{i, k(i)}\right)$ for all $i \in \mathbb{N}$ with $i<k(i)$, is an element of $H_{\mathbb{N}}$. Likewise, since $m_{k(i)}, n_{i, k(i)} \in N_{k(i)}^{*} \cup\left\{m_{k(i)}\right\}$, we have that $h_{f} q h_{f}^{-1} \in H_{\mathbb{N}}$. Therefore $p, q \in\left\langle H_{\mathbb{N}}, g_{f}, h_{f}\right\rangle$. Hence, as $p\left(m_{k(i)}\right)=m_{k(i)}$ and $q\left(m_{i}\right)=m_{i}$ for all $i \in \mathbb{N}$ with $i<k(i)$, it follows that $t=p q p \in\left\langle H_{\mathbb{N}}, g_{f}, h_{f}\right\rangle$, as required.

(iii). It suffices to prove that $\operatorname{rank}\left(S_{\infty}: H_{2}\right)=\mathfrak{c}$ and $\operatorname{rank}\left(S_{\infty}:\left\{1_{\mathbb{N}}\right\}\right)=\mathfrak{c}$. Clearly, $\operatorname{rank}\left(S_{\infty}:\left\{1_{\mathbb{N}}\right\}\right)=\mathfrak{c}$, as $\left\{1_{\mathbb{N}}\right\}$ is countable. We prove that $\operatorname{rank}\left(S_{\infty}\right.$ : $\left.H_{2}\right)=\mathfrak{c}$ using the notion of relative ranks for semigroups. Replacing 'the group generated by' with 'the semigroup generated by' in the definition, it is clear what we mean by the relative rank of a subset of a semigroup. If $\operatorname{rank}\left(S_{\infty}: H_{2}\right)<\mathfrak{c}$, then there exists $U \subseteq S_{\infty}$ with $|U|<\mathfrak{c}$ and $\left\langle H_{2}, U\right\rangle=S_{\infty}$. Hence $S_{\infty}$ equals the semigroup generated by $H_{2}$ and $U \cup U^{-1}$. By [7, Theorem 3.3], there exist $f, g \in \mathbb{N}^{\mathbb{N}}$ such that the semigroup generated by $S_{\infty} \cup\{f, g\}$ is $\mathbb{N}^{\mathbb{N}}$. Thus the semigroup relative rank of $H_{2}$ in $\mathbb{N}^{\mathbb{N}}$ is at most $\left|U \cup U^{-1} \cup\{f, g\}\right|$ and, in particular, it is strictly less than $\mathfrak{c}$. Hence, by the contrapositive, to prove that $\operatorname{rank}\left(S_{\infty}: H_{2}\right)=\mathfrak{c}$ it suffices to show that the semigroup relative rank of $H_{2}$ in $\mathbb{N}^{\mathbb{N}}$ is $\mathfrak{c}$.

Let $A$ be a subset of $\mathbb{N}^{\mathbb{N}}$ such that $H_{2}$ and $A$ generate $\mathbb{N}^{\mathbb{N}}$ as a semigroup. Seeking a contradiction, assume that $|A|<\mathfrak{c}$. The semigroup $\mathbb{N}^{\mathbb{N}}$ is the union of the sets

$$
B_{\left(a_{0}, a_{1}, \ldots, a_{m}\right)}=\left\{h_{m+1} a_{m} h_{m} \cdots h_{1} a_{0} h_{0}: h_{0}, h_{1}, \ldots, h_{m+1} \in H_{2}\right\}
$$

over all tuples $\left(a_{0}, a_{1}, \ldots, a_{m}\right) \in A^{m+1}$ and all $m \in \mathbb{N}$.

A subset $F$ of $\mathbb{N}^{\mathbb{N}}$ is called almost disjoint if the set $\{i \in \mathbb{N}: f(i)=g(i)\}$ is finite for all $f, g \in F$. Let $F$ be an almost disjoint subset of $\mathbb{N}^{\mathbb{N}}$ with $|F|=\mathfrak{c}$. The existence of such an $F$ is well-known; see, for example, [10, Theorem 1.3]. Since $|F|=\mathfrak{c}$ and $\left|A \cup A^{2} \cup \cdots\right|<\mathfrak{c}$, there exist $n \in \mathbb{N}$ and $\left(b_{0}, b_{1}, \ldots, b_{n}\right) \in A^{n+1}$ such that $B_{\left(b_{0}, b_{1}, \ldots, b_{n}\right)} \cap F$ is infinite.

By the definition of $\mathrm{H}_{2}$,

$$
\left|\left\{f(i): f \in B_{\left(b_{0}, b_{1}, \ldots, b_{n}\right)}\right\}\right| \leq 2^{n+2}
$$

for all $i \in \mathbb{N}$. Let $N=2^{n+2}$ and let $f_{0}, f_{1}, \ldots, f_{N}$ be distinct elements of $B_{\left(b_{0}, b_{1}, \ldots, b_{n}\right)}$ $\cap F$. Then, since $F$ is a family of almost disjoint functions, there exists $i \in \mathbb{N}$ such that $f_{0}(i), f_{1}(i), \ldots, f_{N}(i)$ are distinct, contradicting (1). 
We do not know a subgroup of $S_{\infty}$ or a subsemigroup of $\mathbb{N}^{\mathbb{N}}$ with an uncountable relative rank not equal to $\mathfrak{d}$ or $\mathfrak{c}$. So, we ask: Does there exist a subgroup $G$ of $S_{\infty}$ or a subsemigroup $S$ of $\mathbb{N}^{\mathbb{N}}$ such that $\operatorname{rank}\left(S_{\infty}: G\right), \operatorname{rank}\left(\mathbb{N}^{\mathbb{N}}: S\right) \notin\{0,1,2, \mathfrak{d}, \mathfrak{c}\}$ ?

\section{REFERENCES}

1. A. Arhangel'skii and M. Tkachenko, Topological groups and related structures, Atlantis Studies in Mathematics, vol. 1, Atlantis Press, Paris, 2008. MR2433295 (2010i:22001)

2. T. Bartoszyński, H. Judah, and S. Shelah, The Cichoń diagram, J. Symbolic Logic 58 (1993), 401-423. MR1233917 (94m:03077)

3. G. M. Bergman and S. Shelah, Closed subgroups of the infinite symmetric group, Algebra Universalis 55 (2006), 137-173. MR2280223(2008a:20005)

4. P. J. Cameron, Permutation groups, London Mathematical Society Student Texts, vol. 45, Cambridge University Press, Cambridge, 1999. MR.1721031 (2001c:20008)

5. J. Cichoń, J. D. Mitchell, and M. Morayne, Generating continuous mappings with Lipschitz mappings, Trans. Amer. Math. Soc. 359 (2007), 2059-2074. MR2276612 (2007k:54043)

6. F. Galvin, Generating countable sets of permutations, J. London Math. Soc. (2) 51 (1995), 230-242. MR.1325568 (96a:20005)

7. J. M. Howie, N. Ruškuc, and P. M. Higgins, On relative ranks of full transformation semigroups, Comm. Algebra 26 (1998), 733-748. MR1606221 (99b:20099)

8. A. S. Kechris, Classical descriptive set theory, Graduate Texts in Mathematics, vol. 156, Springer-Verlag, New York, 1995. MR 1321597 (96e:03057)

9. A. S. Kechris and C. Rosendal, Turbulence, amalgamation, and generic automorphisms of homogeneous structures, Proc. London Math. Soc. (3) 94 (2007), 302-350. MR 2308230 (2008a:03079)

10. K. Kunen, Set theory, Studies in Logic and the Foundations of Mathematics, vol. 102, NorthHolland Publishing Co., Amsterdam, 1983. MR756630 (85e:03003)

11. H. D. Macpherson and P. M. Neumann, Subgroups of infinite symmetric groups, J. London Math. Soc. (2) 42 (1990), 64-84. MR 1078175 (92d:20006)

12. Z. Mesyan, Endomorphism rings generated using small numbers of elements, Bull. London Math. Soc. 39 (2007), 290-300. MR2323461 (2008f:16066)

13. , Generating self-map monoids of infinite sets, Semigroup Forum 75 (2007), 649-676. MR 2353288 (2008h:20091)

14. J. D. Mitchell, M. Morayne, Y. Péresse, and M. R. Quick, Generating transformation semigroups using endomorphisms of preorders, graphs, and tolerances, Ann. Pure Appl. Logic 161 (2010), 1471-1485.

Mathematics Institute, University of St. Andrews, North Haugh, St. Andrews, Fife, KY16 9SS, SCOTLAND

E-mail address: jdm3@st-and.ac.uk

$U R L$ : tinyurl.com/jdmitchell

Institute of Mathematics and Computer Science, Wroceaw University of Technology, Wybrzeże Wyspiańskiego 27, 50-370 WrocŁaW, Poland

E-mail address: michal.morayne@pwr.wroc.pl

Mathematics Institute, University of St. Andrews, North Haugh, St. Andrews, Fife, KY16 9SS, ScOTLAND

E-mail address: yhp1@st-and.ac.uk 